\title{
The Spike Generation Processes: A Case for Low Level Computation
}

\author{
Tjeerd olde Scheper \\ Department of Computing, Oxford Brookes University, Oxford, OX33 1HX, UK; \\ tvolde-scheper@brookes.ac.uk
}

\begin{abstract}
Summary. Over the last couple of years, it can be said that the focus of the computational aspects of neurons has moved from synaptic weight and firing rate encoding to temporal firing encoding. On the other hand, several elements of these models have been based on some conceptual assumptions that imply relative simple dynamic behaviour of neuronal membrane activity in an active-passive process. In line with recent advances that have produced a better understanding of the biochemical processes that occur within cells, it is proposed that the processes that are involved in a membrane depolarisation cascade are less static than have been assumed so far. In particular, the possibilities of low level computation at the membrane level need to be explored more extensively. In this chapter some computational properties of the spike generation processes are explored using phenomenological models.
\end{abstract}

Key words: Neuronal membrane, computation, Hindmarsh-Rose, chaotic control, synchronisation.

\subsection{Introduction}

The limited dynamic behaviour that exists within models currently studied for spike generation is partly due to the fact that little is known about the low level interaction of the components that generate the depolarisation cascade. Even though it has been known for a long time that the ionic channel dynamics is voltage gated as well as gated by other mechanisms, the interaction between different transmembrane components and intracellular processes is less well known [1,7]. This may, in part, be attributed to the difficulty of measuring accurately the exact state of individual channels and the difficulty of acquiring information about the subcellular processes that are involved in the spike generation cascade of living cells. The construction of theoretical models of these channels has already produced a large body of knowledge about the conductance behaviour of ionic channels. This knowledge is, however, limited by the underlying assumptions of the models. The stable state of most conductance models, such as the well-known Hodgkin-Huxley system, does not include further dynamical aspects beyond mere responses to global changes in voltage. By developing abstract models of more complex systems with the specific aim to produce systems that are capable of 
some level of computation and comparing the performances with biological systems, a much more detailed understanding of the possible processes may be reached.

\subsection{Membrane Elements as Computational Units}

To achieve a better understanding of the processes involved in the possible computations performed at the molecular level in the neuronal membrane, a membrane computational unit can be defined. A membrane computational unit (MCU) is formed by a collection of ionic channels and other transmembrane proteins that contribute to the formation of a single depolarising spike in a neuronal membrane at that point. The composite elements are not evenly distributed nor is it assumed that they are all in a similar state. Indeed, these states may be responsible for a localised mechanism that may be capable of some types of computation. The advantages of such mechanisms are extensive. A combination of localised membrane-specific computation and global computational activity of the entire neuron will allow a staggering amount of processing elements. Furthermore, each MCU does not have to be specifically defined but can be organised in response to local dynamic behaviour.

Local state changes of the complex interactions of membrane depolarising units have not been considered to be very relevant in the overall theory of neuronal computation. Indeed, an emphasis appears to exist to reduce the membrane components of an electrically active cell to mere conductive elements with the computation solely provided by intercellular communication [1]. This model of cellular activity is based on single and population channel dynamics in an enforced fixed state. However, if one considers that the interaction of all the channel proteins are of an extremely complex nature, as can be derived from the rest of the biochemical pathways [2], it appears to be more likely that localised states and state-induced processing are not only possible but functional.

The biophysical structure of the membrane may be described as a mosaic of ionic channels and other membrane-bound proteins. The biophysical organisation of the neuronal membrane mosaic determines the properties of the membrane, such as conductance [3]. This is depicted schematically in Fig. 25.1 where the octagons represent an ionic channel organisation and the circles molecules that are capable of inducing adaptation. In a static model environment, the adaptation may cause the global neuronal behaviour to change in response to a depolarisation input current. In a temporal and spatially dynamic model the localised adaptation may cause the local environment to change in response to local and global effect. This includes both local adaptation as activity-induced adaptation of the membrane mosaic (Fig. 25.2 and 25.3).

The conductance models, as currently used in systems of computational neurons, are generally based on the original models (or variations of it) as defined according to the Hodgkin-Huxley dynamics $[6,15]$. This describes each ionic channel as a continuous state variable whose dynamic behaviour in time is described by first-order kinetics. Each channel equation has an increasing term and a decreasing term of the conductance dependent state variable $n$, e.g., $d n / d t=\alpha_{n}(V) \times(1-n)-\beta_{n}(V) \times n$. 


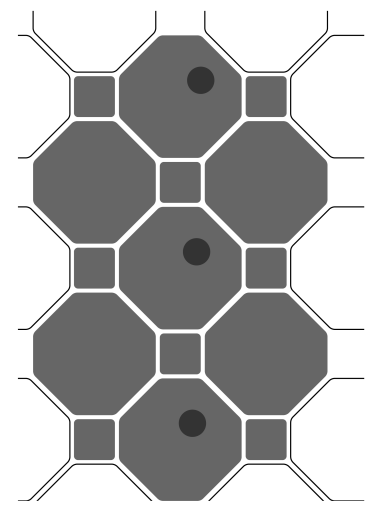

Fig. 25.1. The biophysical mosaic.

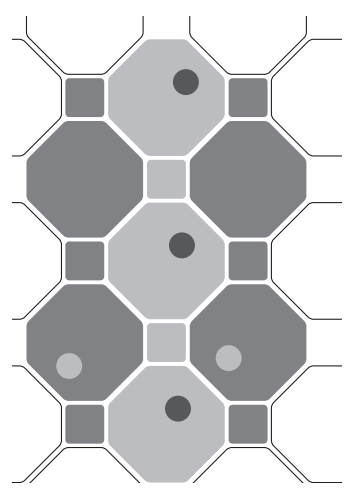

Fig. 25.2. Local response of conductive elements in presence of adaptation.

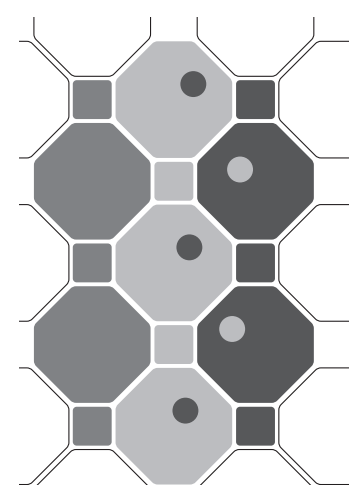

Fig. 25.3. Activity-induced adaptation in nearby conductive elements in a biophysical mosaic.

The activation and inactivation rate functions $\alpha_{n}(V)$ and $\beta_{n}(V)$ were then experimentally determined by varying the voltage. The resulting equations were subsequently derived from the experimental data set by fitting elementary arithmetical operations as functions of the voltage. There are several assumptions made within this model that may now be considered to be too unassuming.

The possible states of the ionic channels can be primarily determined by the conductance but the existence of additional states is not impossible. It could be considered that other states, such as a blocked state, are part of the normal state transitions that occur. The kinetic dynamics of the molecular interactions are rarely of first order within other biochemical reactions and this may be recognised by considering the subdomains and co-proteins of the ionic channel as part of the kinetic equation. The rate of opening and closing as described by the $\alpha(V)$ and $\beta(V)$ functions only describes the channel dynamics but does not explain the mechanisms by which these dynamics have emerged. Furthermore, the functions, as currently used to describe the spike generation process, are incapable of exhibiting other dynamics than fixed state dynamics (by external periodic forcing and similar mechanisms, some more complex dynamic behaviour of the voltage may be produced but these do not change the stable state of the Hodgkin-Huxley system itself).

It can be argued that the localised dynamics exist by virtue of the biochemical processes involved. In other words, the enzymatically controlled chemical reactions and the membrane processes controlled by secondary and tertiary molecular structural changes do allow more complex dynamics to occur. The observation of noisy oscillations is not precluded by this assumption. It may also be considered that if the highly controlled processes that can be found in the biochemical pathways were not present in the proteins involved in the formation of the depolarisation cascade, they would form a complete separate part of the entire biochemical system. An uncontrolled and noisy 
process, such as the depolarisation cascade, is simply not energetically favourable if by controlling the process, energy can be saved. The next step in process control, in the sense of allowing some depolarising currents to result in a spike or of blocking such currents locally, seems to be a mere optimisation of the complete controlled process. True localised computation may then follow readily and it can then be possible to define a spatially and dynamically bound unit which forms the minimally required computational element or MCU.

Ideally, one would like to describe all processes and molecules involved at the biophysical level and study the system using the physical properties of those processes. The complexity of such a modelling system is of very high order and, by assuming that the underlying computational process is not dependent on unique physical circumstances, the use of more phenomenological models is justified with the aim of understanding what is required to achieve some particular computational process.

\subsection{Membrane Computational Unit Model}

With the aim of simulating computational processes within an MCU, several biologically relevant phenomenological models are combined. Each model of an MCU has at least two different components that act together to produce a system which is capable of complex emergent behaviour. One is a spike generation component and the other a controlled chaotic drive component. The spike formation component has been derived from the Hindmarsh-Rose (HR) model [5] but includes a fourth slow recurrent equation which represents the slow calcium exchange between intracellular stores and the cytoplasm [10]. This makes the modified HR model more like a chaotic Hodgkin-Huxley $(\mathrm{HH})$ model of stomatogastric ganglion neurons [10]. The four-dimensional HR can therefore be accurately interpreted as a phenomenological model of the conductance-based HH model. The parameter values for the fourdimensional Hindmarsh-Rose (HR4) model are $a=1, b=3, c=1, d=1, e=1$, $f=5, g=0.0275, u=0.00215, s=4, v=0.001, k=0.9573, r=3.0, m=1$, $n=1$ and rest potential $x_{0}=1.605$. The variable $I$ represents the input to the unit which can be external square input pulses or input from other units. With these parameter values the model is stable in the resting potential but shows low dimensional chaos in the bursting patterns [10].

$$
\begin{aligned}
\frac{d x}{d t} & =a y+b x^{2}-c x^{3}-d z+I \\
\frac{d y}{d t} & =e-f x^{2}-m y-g w \\
\frac{d z}{d t} & =u\left(s\left(x+x_{0}\right)-n z\right) \\
\frac{d w}{d t} & =v(r(y+l)-k w)
\end{aligned}
$$

It is also possible to add another inactivation current which competes with the third current to return the system to the equilibrium state. The third equation of the 
HR4 model (25.3) is then complemented with a fifth equation resulting in the fivedimensional Hindmarsh-Rose model HR5. The effect of the faster inactivation current $z_{f}$ (25.8), compared to the slower inactivation current as used in HR4, is that the system tends to burst less. The faster current makes the system return faster towards the equilibrium where only a larger (re)activation current can cause the system to burst. In the MCU model, the HR5 system allows the temporal separation of spikes by increasing the refractory period. Parameter values for (25.8) are $s_{f_{1}}=8, s_{f_{2}}=1, n_{f}=4$ and the parameter $d_{f}=0.5$ in (25.5). The parameters $s_{s}=4$ and $n_{s}=1$ have the same value as the equivalent parameters in (25.3).

$$
\begin{aligned}
\frac{d x}{d t} & =a y+b x^{2}-c x^{3}-d_{s} z_{s}-d_{f} z_{f}+I \\
\frac{d y}{d t} & =e-f x^{2}-m y-g w \\
\frac{d z_{s}}{d t} & =u\left(s_{s}\left(x+x_{0}\right)-n_{s} z_{s}\right) \\
\frac{d z_{f}}{d t} & =u\left(\left(s_{f_{1}}\left(x+x_{0}\right)-s_{f_{2}} x^{2}\right)-n_{f} z_{f}\right) \\
\frac{d w}{d t} & =v(r(y+l)-k w)
\end{aligned}
$$

Connecting these HR models will result in different types of behaviour, such as stable periodic and chaotic synchronised and unsynchronised behaviour [10]. These depend on continuous, relatively large, inputs to the model. It is possible to make continuous connections of HR4 models that change the chaotic spike bursting into slow oscillations [12]. However, the system need not be purely chaotic to make use of some of the properties of chaotic systems, such as control and synchronisation. A controlled chaotic system is a system which is inherently chaotic but is limited to a controlled state such that the resulting dynamic behaviour is indistinguishable from periodic behaviour [14]. For some types of control, such as rate control and Ott-Grebogi-Yorke (OGY) control $[8,14]$, the chaotic system is only perturbed into the unstable periodic orbit during very small periods of its evolution. Outside the control period, the controlled system is still capable of synchronisation [9]. This feature allows the use of a stable controlled period generated by a controlled chaotic system to synchronise to another system even if they have different periods.

To introduce the required controlled chaotic behaviour in either of the HR models, a scaled and inverted Rössler system has been used [13]. This is necessary because the normal Rössler model has a different time scale from the HR4 model. As can be seen in Table 25.1, the scaled variables are proportional to the normal Rössler parameter values. It is possible to map the time scale of the modified Rössler (R3) model to fit the time scale of the HR4 model and use the R3 system to generate patterns. In addition to the scaling, the $u_{r}$ variable has been inverted to enable the convenient use of this variable as the drive for the HR4 model.

$$
\frac{d x_{r}}{d t}=-b_{r} y_{r}-d_{r} u_{r}
$$


Table 25.1. Scaled parameter values compared to normal Rössler model values.

\begin{tabular}{|c|c|c|}
\hline Parameter & Normal value & Scaled value \\
\hline$a_{r}$ & $\frac{1}{5}$ & $\frac{1}{75}$ \\
$b_{r}$ & 1 & $\frac{1}{15}$ \\
$c_{r}$ & 1 & $\frac{1}{15}$ \\
$d_{r}$ & 1 & $\frac{1}{50}$ \\
$k_{r}$ & 5.7 & -0.57 \\
$w_{r}$ & $\frac{1}{5}$ & $-\frac{1}{75}$ \\
$p_{r}$ & 1 & -1 \\
\hline
\end{tabular}

$$
\begin{aligned}
& \frac{d y_{r}}{d t}=c_{r} x_{r}+a_{r} y_{r} \\
& \frac{d u_{r}}{d t}=p_{r} u_{r} x_{r}+k_{r} u_{r}+w_{r}
\end{aligned}
$$

AU: Why mix decimals and fractions in Table 25.1?
The R3 system is controlled into an unstable periodic orbit using a chaotic rate control mechanism [8]. This mechanism allows the system to exhibit different periodic orbits by limiting the rate of change of equation (25.12). The rate control variable $\sigma$ is only different from 1 if the variables $x$ and $u$ are diverging rapidly, i.e., when the chaotic manifold is stretching or folding. Equation (25.12) is modified to (25.14) as shown below. The rate control parameter $\mu$ determines the strength of the rate limiting function and the parameter $\xi$ can have different values but is usually $-2 \leq \xi<0$. This chaotic control mechanism is very effective at stabilising different unstable periodic orbits, but not for any given value of $\mu$ and $\xi$. Typically used values are $\mu=6$ and $\xi=-1$ or $\xi=-2$.

$$
\begin{aligned}
\sigma(x, u) & =e^{\frac{\xi(x u)}{(u+x+\mu)}} \\
\frac{d u_{r}}{d t} & =\sigma\left(x_{r}, u_{r}\right) p_{r} u_{r} x_{r}+k_{r} u_{r}+w_{r} .
\end{aligned}
$$

The controlled chaotic system is now connected to the four-dimensional HindmarshRose system via the HR4 $z$ variable. Equation (25.3) is subsequently modified to become

$$
\frac{d z}{d t}=u\left(s\left(x+x_{0}\right)-q u_{r} z\right)
$$

Because the $u_{r}$ variable of the scaled Rössler R3 system is always negative, the parameter $q$ in equation (25.15) is negative: $q=-12$.

Finally, different HR-type systems may be connected electrically by summation of the two main currents. It is possible to extend this to all currents but this does not seem to have a significant effect on the qualitative behaviour of the two connected systems. 
By adding the total differences in activity of the HR models' $x$ and $y$ variables as follows, depending on the choice of $\alpha$, different dynamic behaviour will result:

$$
\begin{gathered}
I_{i}(x)=\alpha_{i}(x) \sum_{j}\left(x_{j}-x_{i}\right) \\
I_{i}(y)=\alpha_{i}(y) \sum_{j}\left(y_{j}-y_{i}\right) .
\end{gathered}
$$

For example, for $\alpha(x), \alpha(y)>0$ the resulting HR system will act as a logical AND of the input spikes. With $\alpha(x)=0$ and $\alpha(y)>0$, the resulting HR system acts as an AND-NOT gate, i.e., it is only active if one of the inputs has produced a spike but not if both spike together and not if only the other input has a spike. This may be used to detect both coincidental spikes as well as single spikes from one source only.

\subsection{SyncMCU}

The different computational elements described may be combined to construct an ensemble of computational elements capable of solving computational problems. For example, consider Fig. 25.4 where five computational units are linked. Here, units HR4R3-1 and 2 are made from four-dimensional HR systems, driven by a controlled scaled Rössler system R3. Unit HR5-AND consists of a single HR5 system, without a controlled chaotic drive, but electrically connected to units HR4R3-1 and 2 using (25.16) and (25.17) with $\alpha(x), \alpha(y)>0$. Unit HR4-ANDNOT consists of a four-dimensional HR4 system but with a scaled R3 drive. It receives input from units HR4R3-1 and 2 but with $\alpha(x)=0$ and $\alpha(y)>0$. Lastly, unit HR4 is a normal HR4 system without an R3 drive, that only receives input from unit HR5-AND. All the R3 drive systems are controlled in the same unstable periodic orbit but the driving scalar

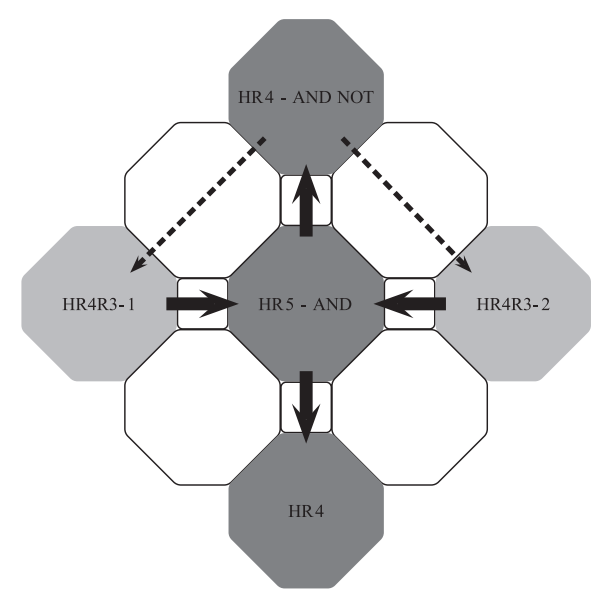

Fig. 25.4. Schematic representation of the SyncMCU model. 
is small such that by itself it does not cause the system to fire. The R3 systems may therefore act as a localised subcellular clock that can be in or out of sync with other units.

The configuration shown in Fig. 25.4 may act as a detector of desynchronisation of two input signals. Given an additional external input to the units HR4R3-1 and 2, which are combined in unit HR5-AND and then passed on to unit HR4, the unit HR4ANDNOT will detect if unit HR4R3-2 fires but HR4R3-1 does not. Note that if they both fire, HR4-ANDNOT does not fire unless it has fired recently. We can now use this to attempt to synchronise unit HR4R3-2 with unit HR4R3-1 even if they have completely different periods.

To enable unit HR4-ANDNOT to synchronise the units HR4R3-1 and 2, a synchronisation function is defined as

$$
\frac{d S}{d t}=\kappa_{1}\left(x_{r}^{1}-x_{r}^{2}\right) \theta(x)-\kappa_{2} S,
$$

where $\kappa_{1}$ and $\kappa_{2}$ are the growth and decay parameters, and $x_{r}^{n}$ are the $x_{r}$ variables of the controlled chaotic scaled Rössler systems of the units that are synchronised. The function $\theta(x)$ is a threshold function on the $x$ variable of the HR4 system of the unit HR4-ANDNOT. Parameters for (25.18) are $\kappa_{1}=-0.75, \kappa_{2}=0.5$ with the threshold set at -0.5 .

\subsection{Results}

In Fig. 25.5 and 25.6 can be found the results of the SyncMCU model. The model is integrated using different time steps and verified with several numerical integrators, such as the fifth-order Runge-Kutta and Prince-Dormand Runge-Kutta integrators, which all produced qualitatively similar results. Because the model cannot start from an a priori established initial stable state, the model is run for 5000 time steps without chaotic control to allow the Rössler model to reach the domain of its strange attractor. At time step 5000, the chaotic control is enabled, resulting in subthreshold activity which ensures that the model is in a stable periodic orbit before external input is presented. At time step 10000, the external input is enabled which generates pulses of width 10 with period 290 for HR4R3-1 and period 400 for HR4R3-2.

In both Fig. 25.5 and 25.6, the $x$ variables of HR4R3-1 and 2 are shown for the first 25000 time steps only. This enables the period, due to the combined effect of the controlled chaotic drive and the external input, to become visible. They are verified to continue in the same multiorbit for very long runs. As can be seen in Fig. 25.5(c) and (d), in the unsynchronised case, units HR5-AND and HR4-ANDNOT show spiking patterns at the combined harmonic input periods. Because the system is responding to the effects of the external input combined with its internal controlled chaotic drive, the emerging patterns appear fairly noisy.

In the synchronised case, as shown in Fig. 25.6(c) and (d), the emerging patterns are corrected by the synchronisation pulses shown in $25.7(b)$ on the internal unit controlled chaotic drive, and the patterns are much less noisy than in the unsynchronised case. By 


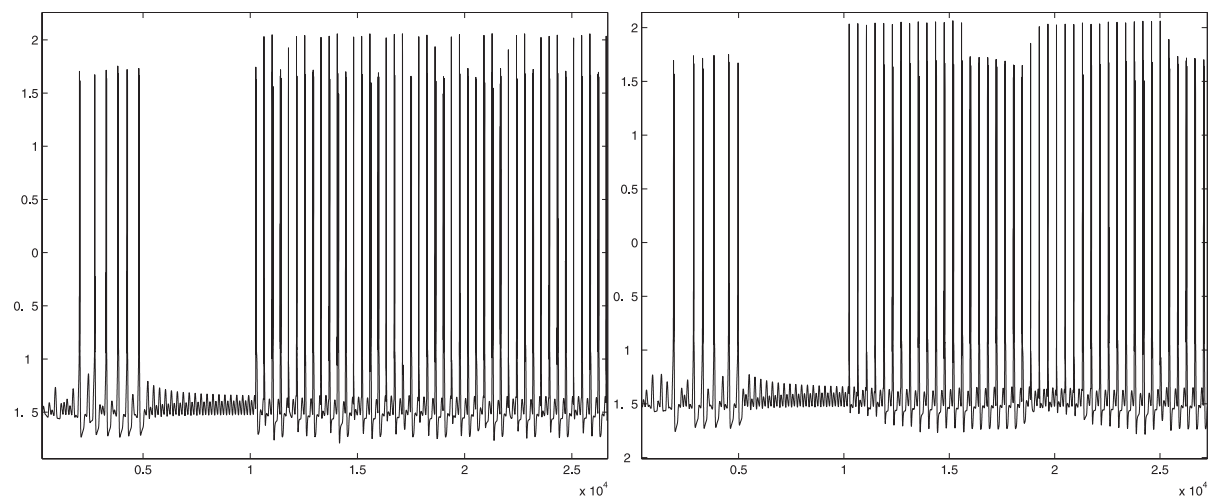

(a) Evolution of $x$ variable of HR4R3-1.

(b) Evolution of $x$ variable of HR4R3-2.
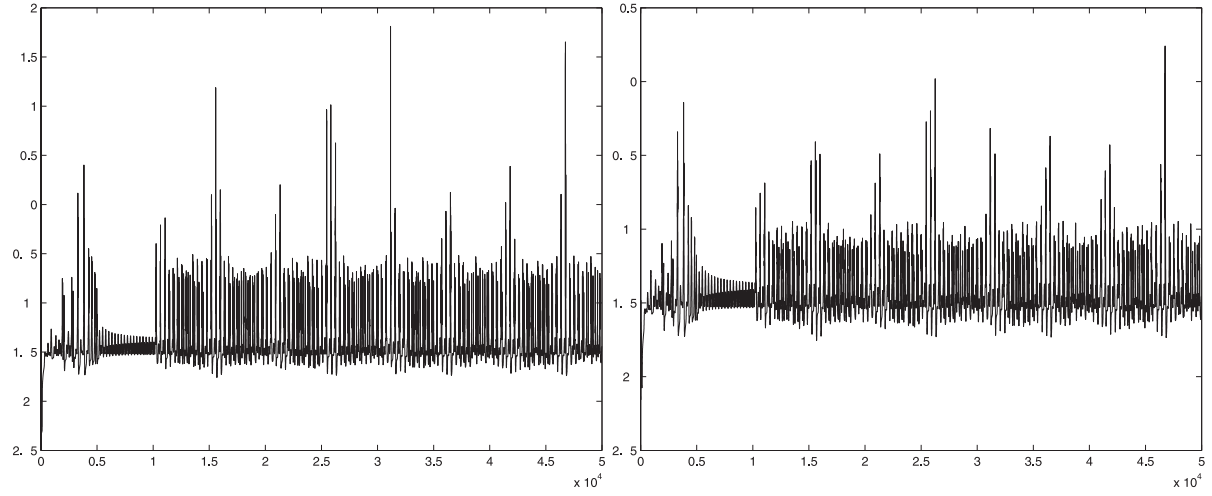

(c) Evolution of $x$ variable of HR5-AND.

(d) Evolution of $x$ of HR4-ANDNOT.

Fig. 25.5. SyncMCU model without synchronisation.

superimposing the synchronised and unsynchronised unit HR4-ANDNOT in Fig, 25.7 (a) the extent of synchronisation correction becomes more clear, indicating that the correction made by the synchronisation function is effective even though the periods of the external input patterns are very much different.

\subsection{Conclusion}

Using a combination of phenomenological models, it may become possible to study computational aspects of neuronal membrane functions. The computational aspects that can be modelled using the MCU paradigm can give indications of biophysical features that may be hidden from the experimentalist at this moment. It can also extend the computational ability of neural and neuronal networks by more distributed computation and the use of simple computational steps to perform important signal processing functions.

The synchronisation model is based on the synchronisation capabilities of the con-

AU: Is the subtitle "(c)" in Fig. 6 OK as changed? 

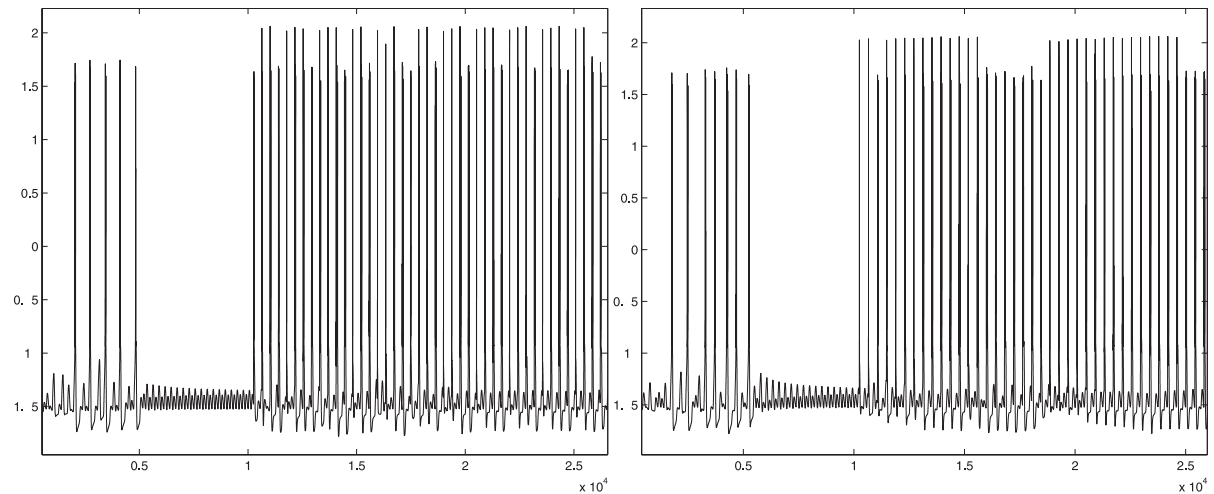

(a) Evolution of $x$ variable of HR4R3-1.

(b) Evolution of $x$ variable of HR4R3-2.
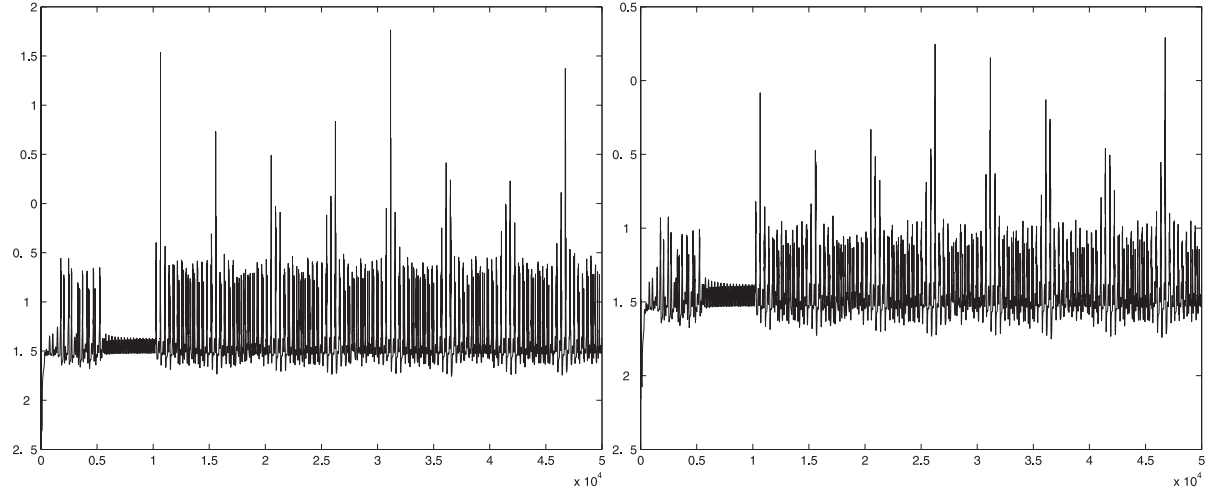

(c) Evolution of $x$ variable of HR5-AND.

(d) Evolution of $x$ of HR4-ANDNOT.

Fig. 25.6. SyncMCU model with synchronisation.

trolled chaotic internal drive. Even though the underlying internal drive is based on a scaled chaotic Rössler model, the resulting system is stable periodic due to the control. The emergent behaviour of the model is due to the interaction of the different periodic external inputs to HR4R3-1 and HR4R3-2 with the stable periodic controlled drive which is summed in HR5-AND and filtered through HR4-ANDNOT, resulting in a synchronisation pulse to HR4R3-2. This, finally, causes the internal controlled drive to synchronise to the difference between the inputs to HR4R3-1 and HR4R3-2. Introducing white Gaussian noise in the external input frequencies does not prevent the system from synchronising although more correcting synchronisation pulses are needed (not shown).

Results from conceptual models as presented may provide indications to identify localised computation in the neuron. Recent experimental results in the subunits of thin dendrites [11] indicate the possible important role of spatial compartmentalisation. Additionally, experimental results, obtained by looking at learning by geometrical shape changes of dendritic spikes, have shown that a single spike event is capable of modulation of the signal transmission [4]. The MCU paradigm may provide a computational 


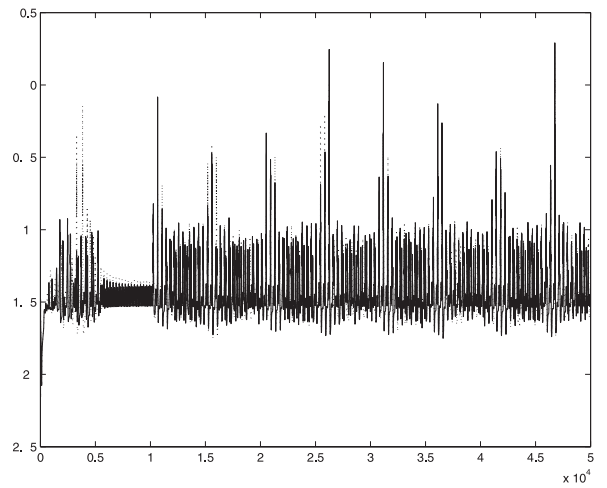

(a) Evolution of $x$ variables of HR4-ANDNOT, solid line synchronised, dotted line unsynchronised.

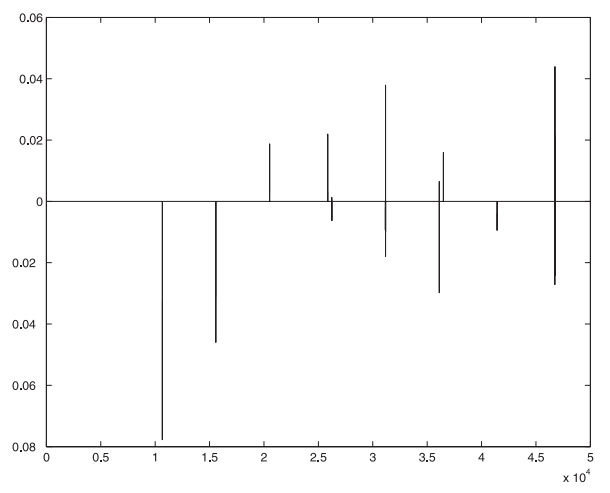

(b) Synchronisation pulses of the synchronisation function $S$ when synchronising unit HR4R3-2.

Fig. 25.7. SyncMCU model with synchronisation.

framework from which the computational abilities of dendritic structures can be studied.

\section{Acknowledgments}

Many thanks to Dr Nigel Crook and Professor Cyriel Pennartz for their valuable contributions and discussion.

\section{References}

1. Dayan, P., Abbott, L.F.: Theoretical Neuroscience, Computational and Mathematical Modeling of Neural Systems. MIT Press Cambridge, MA (2001).

2. Fell, D.A.: Understanding the Control of Metabolism. Portland Press, London (1997).

3. Graham, L.J., Kado, R.T.: The Handbook for Brain Theory and Neural Networks. Chapter: The neuron's biophysical mosaic and its computational relevance. MIT Press, Cambridge, MA, 2nd edition (2002), 170-175.

4. Herzog, A., Spradvedlyvyy, V., Kube, K., Schnabel, R., Korkotian, E., Braun, K., Michaelis, B.: Learning by geometrical shape changes of dendritic spikes. In: Proceedings of European Symposium on Artificial Neural Networks 2004 (2004), 385-390.

5. Hindmarsh, J.L., Rose, R.M.: A model of neuronal bursting using three coupled first order differential equations. Proc. R. Soc. Lond., B 221, 87-102 (1984).

6. Hodgkin, A.L., Huxley, A.F.: A quantitative description of membrane current and its application to conduction and excitation in nerve. J. Phys., 117, 500-544 (1952).

7. Maass, W., Bishop, C.M. (eds.): Pulsed Neural Networks. MIT Press, Cambridge, MA (2001)

8. olde Scheper, T.: Rate control of chaotic systems. in preparation (2006).

9. Pecora, L.M., Caroll, T.L.: Synchronization in chaotic systems. Phys. Rev. Lett., 64, 821824 (1989)
AUCan you provide slightly different titles for Figs. 6 and 7? They are exactly the same.

AU: Can you update Ref. 8? 
10. Pinto, R.D., Varona, P., Volkovskii, A.R., Szucs, A., Abarbanel, H.D.I., Rabinovich, M.I.: Synchronous behavior of two coupled electronic neurons. Phys. Rev. E, 62 (N2 PTB), 2644 2656 (2000).

11. Polsky, A., Mel, B.W., Schiller, J.: Computational subunits in thin dendrites of pyramidal cells. Nature Neuroscience, 7, 621-627 (2004).

12. La Rosa, M., Rabinovich, M.I., Huerta, R., Abarbanel, H.D.I, Fortuna, L.: Slow regularization through chaotic oscillation transfer in a unidirectional chain of Hindmarsh-Rose models. Phys. Lett. A, 266, 88-93 (2000).

13. Rössler, O.E.: An equation for continuous chaos. Phys. Lett., 57A, 397-398 (1976).

14. Schuster, H.G. (ed): Handbook of Chaos Control. Wiley-VCH Verlag GmbH (1999).

15. Traub, R.D.: Neuronal Networks of the Hippocampus. Cambridge University Press, London (1991). 